\title{
Choroidal thinning in high myopia measured by optical coherence tomography
}

This article was published in the following Dove Press journal:

Clinical Ophthalmology

13 May 2013

Number of times this article has been viewed

\section{Yasushi Ikuno \\ Satoko Fujimoto \\ Yukari Jo \\ Tomoko Asai \\ Kohji Nishida}

Department of Ophthalmology, Osaka University Graduate School of Medicine, Osaka, Japan
Correspondence: Yasushi lkuno Department of Ophthalmology - E7, Osaka University Graduate School of Medicine, 2-2 Yamadaoka, Suita, Japan Tel +8I 668793456

Fax +8I 668793458

Email ikuno@opththal.med.osaka-u.ac.jp
Purpose: To investigate the rate of choroidal thinning in highly myopic eyes.

Patients and methods: A retrospective observational study of 37 eyes of 26 subjects (nine males and 17 females, mean age $39.6 \pm 7.7$ years) with high myopia but no pathologies who had undergone spectral domain optical coherence tomography and repeated the test 1 year later ( $1 \pm 0.25$ year) at Osaka University Hospital, Osaka, Japan. Patients older than 50 years with visual acuity worse than $20 / 40$ or with whitish chorioretinal atrophy involving the macula were excluded. Two masked raters measured the choroidal thicknesses (CTs) at the fovea, $3 \mathrm{~mm}$ superiorly, inferiorly, temporally, and nasally on the images and averaged the values. The second examination was about 365 days after the baseline examination. The $\mathrm{CT}$ reduction per year (CTRPY) was defined as (CT 1 year after - baseline CT)/days between the two examinations $\times 365$. The retinal thicknesses were also investigated.

Results: The CTRPY at the fovea was $-1.0 \pm 22.0 \mu \mathrm{m}$ (range -50.2 to 98.5 ) at the fovea, $-6.5 \pm 24.3 \mu \mathrm{m}$ (range -65.8 to 90.2 ) temporally, $-0.5 \pm 22.3 \mu \mathrm{m}$ (range -27.1 to 82.5 ) nasally, $-9.7 \pm 21.7 \mu \mathrm{m}$ (range -40.1 to 60.1 ) superiorly, and $-1.4 \pm 25.5 \mu \mathrm{m}$ (range -85.6 to $75.2)$ inferiorly. There were no significant differences in the CTRPY at each location $(P=0.34)$. The CT decreased significantly $(P<0.05)$ only superiorly. The superior CTRPY was negatively correlated with the axial length $(P<0.05)$. The retinal thickness at the fovea did not change. Stepwise analysis for CTRPY selected axial length $\left(P=0.04, R^{2}=0.13\right)$ and age $(P=0.08$, $\left.R^{2}=0.21\right)$ as relevant factors

Conclusions: The highly myopic choroid might gradually thin and be affected by many factors. Location and axial length are key factors to regulate the rate of choroidal thinning in highly myopic eyes. Overall choroidal thickness was not found to change significantly. Longer follow-ups are needed.

Keywords: high myopia, choroid, optical coherence tomography, age

\section{Introduction}

Myopia is characterized by elongation of the axial length (AL). The mechanism is unknown, but several studies have reported that the process of AL elongation is slow and stable, except at the onset of myopia, when the AL has the fastest rate of change. ${ }^{1}$ Excessive AL elongation leads to various kinds of high-myopia-related complications, such as lacquer crack formation, choroidal neovascularization, and chorioretinal atrophy. ${ }^{2}$ Some macular diseases, such as macular holes and foveoschisis accompany high myopia and require vitreoretinal surgeries. ${ }^{3,4}$

Chorioretinal atrophy, augmented by choroidal thinning, is the major visionthreatening complication in high myopia. Histologic studies have reported that atrophy is thought to result from large- and small-vessel obstruction and replacement 
with fibrous tissue. ${ }^{5}$ Choroidal atrophy ultimately leads to photoreceptor cellular death and consequent loss of central vision, because of interruption of the oxygen and nutrition supply. ${ }^{6}$

Recent optical coherence tomography (OCT) technologies have allowed study of choroidal thinning. ${ }^{7,8}$ Generally, there are two OCT systems, ie, enhanced depth imaging $^{9}$ and high-penetration $\mathrm{OCT},{ }^{10}$ for observing the deep ocular tissues. Myopia facilitates deep choroidal imaging using spectral domain OCT, but without enhanced depth imaging or high-penetration OCT, because of choroidal thinning and consequent stronger signal from the deep choroid. ${ }^{7}$ The refractive error (RE), AL, and especially age are critical for evaluation of choroidal thickness (CT). ${ }^{7,8}$

The goal of the current study was to determine how fast the choroid thins in highly myopic eyes. We measured changes in choroidal thickness in otherwise healthy highly myopic eyes at baseline and 1 year later using OCT.

\section{Materials and methods Patients}

We retrospectively reviewed the records of 591 eyes of 301 subjects who were referred to our High Myopia Clinic at Osaka University Hospital, Osaka, Japan, from September 2007 to April 2010, and chose the subjects with normal high myopia who met the inclusion criteria and did not meet the exclusion criteria. The inclusion criteria were the presence of high myopia with a spherical equivalent RE greater or equal to -6 diopters; no apparent macular abnormalities, such as choroidal neovascularization, chorioretinal atrophy, myopic foveoschisis, macular hole, and others; no peripheral myopic degenerations; and no history of intraocular surgeries. The exclusion criteria were patient age older than 50 years, visual acuity worse than 20/40 because of poor fixation, and focal or spotty whitish chorioretinal atrophy involving the macula. Finally, 37 eyes of 26 subjects (nine men, 17 women) with normal high myopia who underwent spectral domain OCT at baseline and $1 \pm 0.25$ year later were selected. The internal review board approved the study protocol, and all patients provided written informed consent for participation in the study. The study adhered to the tenets of the Declaration of Helsinki.

\section{Examinations}

The choroid was visualized using Cirrus OCT (Carl Zeiss Meditec, Jena, Germany). The scan patterns included the macular cube $512 \times 128$ combo protocol. This scan protocol generates a cube of data through a $6 \mathrm{~mm}$ square grid by acquiring a series of 128 horizontal scan lines comprising 512 A scans. The choroidal thicknesses at the fovea and $3 \mathrm{~mm}$ superiorly, inferiorly, temporally, and nasally were measured initially and 1 year later and analyzed by macular thickness analysis mode. Two masked raters independently measured the choroidal thicknesses, defined as the vertical distance between the retinal pigment epithelium and the chorioscleral interface, and recorded the mean value. The patients underwent a second examination about 365 days later. The choroidal thickness reduction per year (CTRPY) was calculated, which was defined by the following formula:

$$
\begin{aligned}
\text { CTRPY }= & (\text { CT } 1 \text { year after }-\mathrm{CT} \text { at baseline }) / \text { days } \\
& \text { between the two examinations } \times 365 \text {. }
\end{aligned}
$$

The averaged foveal retinal thicknesses ( $1 \mathrm{~mm}$ circle by cube scan with Cirrus OCT) were also investigated initially and 1 year later. The AL was measured using the IOLMaster (Carl Zeiss Meditec), the intraocular pressure (IOP) using a noncontact tonometer (CT-90 A; Topcon, Tokyo, Japan), and the RE using an Auto Refractometer (ARK-530; Nidek, Gamagori, Japan). To determine the factors associated with choroidal thickness and CTRPY, we investigated sex, age, AL, RE, and IOP.

\section{Statistical analysis}

The data were analyzed using JMP version 8.0 (SAS Institute, Cary, NC, USA). One-way analysis of variance and the paired $t$-test were performed as appropriate. $P<0.05$ was considered significant. Stepwise analysis was used to select the most influential factors.

\section{Results}

\section{Patient demographic data}

This study included 37 eyes of 26 subjects (nine men, 17 women) (mean patient age $39.6 \pm 7.7$ years [mean \pm standard deviation]). The mean ( \pm standard deviation) baseline IOP was $16.2 \pm 2.5 \mathrm{mmHg}$, mean $\mathrm{RE}-12.0 \pm 3.4$ diopters, and mean AL $29.1 \pm 1.6 \mathrm{~mm}$.

\section{Case report}

A 49-year-old woman with high myopia had visual loss in the right eye and was referred to our clinic. She had undergone vitrectomy and lensectomy for retinoschisis in the right eye. The vision in the right eye improved postoperatively from 20/50 to $20 / 20$, and the retina was reattached. During the follow-up period, OCT was performed in both eyes. Her left eye also was myopic (Figure 1), and best-corrected visual acuity in the left 


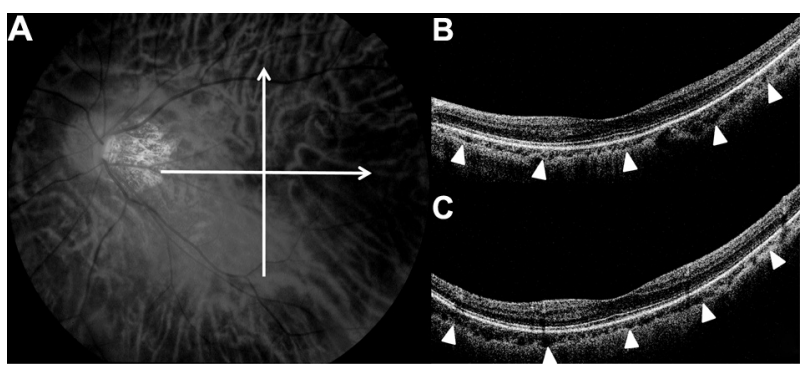

Figure I Color fundus photograph (A) and optical coherence tomographic appearance ([B] horizontal, $[\mathbf{C}]$ vertical at baseline) (right) of the representative case.

Notes: The white bar in the fundus photograph indicates the retinal site at which the optical coherence tomographic images were obtained. We measured the choroidal thickness at the fovea, temporally, nasally, superiorly, and inferiorly at baseline and I year later (arrowheads).

eye was $20 / 20$. The RE was -10.0 diopters at baseline, and the AL was $29.03 \mathrm{~mm}$. The OCT images showed choroidal thinning. The foveal thickness was $83 \mu \mathrm{m}$, temporal thickness $139 \mu \mathrm{m}$, nasal thickness $62 \mu \mathrm{m}$, superior thickness $91 \mu \mathrm{m}$, and inferior thickness $164 \mu \mathrm{m}$ (Figure 1). One year later, the AL had elongated to $29.14 \mathrm{~mm}$, and the choroidal thickness had decreased to $65 \mu \mathrm{m}$ at the fovea, $126 \mu \mathrm{m}$ temporally, $49 \mu \mathrm{m}$ nasally, $80 \mu \mathrm{m}$ superiorly, and $155 \mu \mathrm{m}$ inferiorly.

\section{Choroidal thickness}

The mean baseline choroidal thickness was $95.6 \pm 60.7 \mu \mathrm{m}$ (range 16-214) at the fovea, $121.8 \pm 64.0 \mu \mathrm{m}$ (range 21-265) temporally, $61.1 \pm 33.1 \mu \mathrm{m}$ (range $0-114$ ) nasally, $125.9 \pm 68.6 \mu \mathrm{m}$ (range 22-300) superiorly, and $109.1 \pm 60.8 \mu \mathrm{m}$ (range 24-265) inferiorly. The mean choroidal thickness after 1 year was $91.4 \pm 57.9 \mu \mathrm{m}$ (range 15-223) at the fovea, 117.2 $\pm 65.5 \mu \mathrm{m}$ (range 19-280) temporally, $61.5 \pm 36.5 \mu \mathrm{m}$ (range 0-145) nasally, $121.8 \pm 61.4 \mu \mathrm{m}$ (range 28-277) superiorly, and $110.0 \pm 60.3 \mu \mathrm{m}$ (range 16-224) inferiorly. The choroidal thickness tended to decrease after 1 year at all locations; however, the differences did not reach significance in the four locations ( $P=0.10$ at the fovea, $P=0.17$ temporally, $P=0.84$ nasally, and $P=0.74$ inferiorly, by the paired $t$-test). Only the decrease in the superior choroidal thickness was significant ( $P=0.013$, by paired $t$-test).

\section{Choroidal thickness reduction} rate per year

CTRPY values were $-1.0 \pm 22.0 \mu \mathrm{m}$ (range -50.2 to 98.5) at the fovea, $-6.5 \pm 24.3 \mu \mathrm{m}$ (range -65.8 to 90.2) temporally, $-0.5 \pm 22.3 \mu \mathrm{m}$ (range -27.1 to 82.5 ) nasally, $-9.7 \pm 21.7 \mu \mathrm{m}$ (range -40.1 to 60.1 ) superiorly, and $-1.4 \pm 25.5 \mu \mathrm{m}$ (range -85.6 to 75.2 ) inferiorly.
There were no significant differences in the CTRPY at each location ( $P=0.34$, by analysis of variance). The superior CTRPY was significantly correlated with $\mathrm{AL}(P=0.02)$. The greater CTRPY was, the shorter the AL. There were no significant relationships between the CTRPY at all other locations and baseline factors.

\section{Stepwise regression analysis for CTRPY}

Stepwise analysis for CTRPY identified AL $(P=0.04$, $\left.R^{2}=0.13\right)$ as a factor significantly associated with thinning of the choroid. Age $\left(P=0.08, R^{2}=0.21\right)$ was a second associated factor, though not a significant one.

\section{Retinal thickness}

The mean retinal thicknesses were $258.7 \pm 24.6 \mu \mathrm{m}$ at baseline and $258 \pm 27.1 \mu \mathrm{m}$ after 1 year. There was no significant difference between baseline and 1 year $(P=0.65)$.

\section{Discussion}

We investigated the choroidal thickness changes over 1 year in young, otherwise healthy, highly myopic eyes. As of February 2013, no articles describing a time course of choroidal thickness within the same individuals were found in a PubMed search with the following keywords: choroid, myopia, and optical coherence tomography. To the best of our knowledge, the current study was the first to report the time course of the changes in choroidal thickness in highly myopic eyes using OCT. Choroidal thinning or atrophy was challenging to quantify using conventional methods before the introduction of OCT. The ability to measure choroidal thickness using OCT has enabled quantitative evaluation of the degree of chorioretinal atrophy. It has been found that retinal and choroidal thicknesses are well correlated between instruments and that higher reliability and reproducibility are expected for choroidal thickness measurements, despite having higher morphologic interindividual variations. ${ }^{11}$ Our study also seems to have high reliability and reproducibility in choroidal thickness measurements. The current study showed that choroidal thickness decreased significantly within 1 year superiorly in all cases evaluated. Though the sample size was too small and the follow-up time too short for this conclusion to be generalized for all highly myopic eyes, choroidal thickness might change on a certain time course at least in some locations, and the rate of choroidal thinning might be asymmetrical. Also, the thicker choroid would possibly lose the greater degree of thickness at a time in the superior area. The extremely thin choroid in highly myopic eyes might have hit bottom and the thinning rate might have been slowed down. 
Pathological myopia is progressive in many cases, and $\mathrm{AL}$ is a parameter that indicates the degree of myopia. As pointed out, it is well known to magnify the B scan in highly myopic eyes. ${ }^{12,13}$ However, this is an intraindividual comparison. Saka et al reported that AL is estimated to change by $0.13 \mathrm{~mm}$ per 2 years, ${ }^{14}$ and using the compensation formula, ${ }^{12}$ the location might shift by $3 \mu \mathrm{m}$ per year. The lateral resolution of OCT is $20 \mu \mathrm{m}$. Therefore, though the current study did not examine AL elongation, the shifting seems to be much less compared with this size.

In the current study, only patients under 50 years old were included. Older patients normally have pathologies at the macula that often misbehave to fixate the target, which might be a bias of the study. We investigated the incidence of this before this study, and our preliminary investigation found the incidence was much lower in patients less than 50 years. In addition, it is well known that the choroid becomes thinner in older patients. ${ }^{15,16}$ It was too hard to measure the extremely thin choroid in older highly myopic patients. Thus, we finally decided our inclusion and exclusion criteria.

The mean subfoveal choroidal thickness in the current study was $95.6 \mu \mathrm{m}$, which agreed with previous reports. ${ }^{7,8}$ Choroidal thickness also is affected by aging. ${ }^{15,16}$ Margolis and Spaide reported that regression analysis showed that the subfoveal choroid in normal eyes decreased $15.6 \mu \mathrm{m}$ for each decade of life. ${ }^{15}$ The current study showed that subfoveal choroidal thickness decreased by an average of $1.0 \mathrm{~mm}$ in 1 year. Further and longer study is needed to estimate this decrease. The decreases in choroidal thickness varied among the ocular locations in the current study, from 9.7 superiorly to $0.5 \mathrm{~mm}$ nasally for 1 year. To the best of our knowledge, no studies have reported the time course of the changes in the choroidal thickness outside of the fovea.

In the current study, the nasal quadrant was thinner than the fovea and temporal quadrant, which is in agreement with a previous report. ${ }^{7}$ Choroidal thickness reportedly differed among locations. In normal eyes, the choroid was thicker at the fovea than temporally and nasally, and the temporal and nasal thicknesses were similar to the superior and inferior thicknesses. ${ }^{16}$ Two possible reasons for the relative choroidal thinning nasally and inferiorly in normal eyes are the choroidal watershed and the fetal choroidal fissure, which closes inferiorly at 7 weeks. ${ }^{17}$ However, in myopic eyes, the choroid is very thin. ${ }^{7,8}$ It is reported to be thickest superiorly and thinnest nasally and at the fovea. ${ }^{7} \mathrm{~A}$ watershed zone in the central fovea may lead to more thinning during expansion of the globe. ${ }^{18}$ The current thickness data agreed with these previous studies.
The current study had some limitations, in that it was retrospective and had a small number of subjects. All measurements on the OCT images were done manually, not automatically. This retrospective study lacks such parameters as AL, IOP, and RE after 1 year because of the missing data. We meant to detect the most influential parameters at baseline to choroidal change. The sensitivity of the methodology has limited this study, because vertical resolution of the Cirrus OCT is $6 \mu \mathrm{m}$, and it is impossible to detect very small changes. This study also lacked long-term follow-up, and the changes in choroidal thickness may not occur at the same speed. Although CTRPY may be affected by age, it remains unclear, because older patients were excluded from this study. However, this exclusion might not be suitable, and it is difficult to know if the changes in choroidal thickness are related to the presence of high myopia, the progression of high myopia, or whether it is a normal aging change. Further studies are needed to investigate these limitations.

\section{Acknowledgment}

Presented in part at the Annual Meeting of American Academy of Ophthalmology, October 23, 2011, Orlando, FL, USA.

\section{Disclosure}

We have no financial interest in any of the products mentioned in this manuscript. The authors report no conflicts of interest in this work.

\section{References}

1. Mutti DO, Hayes JR, Mitchell GL, et al. Refractive error, axial length, and relative peripheral refractive error before and after the onset of myopia. Invest Ophthalmol Vis Sci. 2007;48:2510-2519.

2. Curtin BJ, Karlin DB. Axial length measurements and fundus changes of the myopic eye. Am J Ophthalmol. 1971;1:42-53.

3. Siam A. Macular hole with central retinal detachment in high myopia with posterior staphyloma. Br J Ophthalmol. 1969;53:62-63.

4. Takano M, Kishi S. Foveal retinoschisis and retinal detachment in severely myopic eyes with posterior staphyloma. Am J Ophthalmol. 1999;128:472-476.

5. Ohno H. [Electron microscopic studies of myopic retinochoroidal atrophies 1. Choroidal changes]. Folia Ophthalmol Jpn. 1983;43: 1244-1253. Japanese.

6. Matsuo N, Okabe S, Hasegawa E, et al. Report of research committee on chorioretinal degenerations. Tokyo: Ministry of Health and Welfare of Japan; 1978. Japanese.

7. Ikuno Y, Tano Y. Retinal and choroidal biometry in highly myopic eyes with spectral-domain optical coherence tomography. Invest Ophthalmol Vis Sci. 2009;50:3876-3880.

8. Fujiwara T, Imamura Y, Margolis R, Slakter JS, Spaide RF. Enhanced depth imaging optical coherence tomography of the choroid in highly myopic eyes. Am J Ophthalmol. 2009;148:445-450.

9. Spaide RF, Koizumi H, Pozzoni MC. Enhanced depth imaging spectraldomain optical coherence tomography. Am J Ophthalmol. 2008;146: 496-500. 
10. Unterhuber A, Povazay B, Hermann B, Sattmann H, Chavez-Pirson A, Drexler W. In vivo retinal optical coherence tomography at $1040 \mathrm{~nm}$ - enhanced penetration into the choroid. Opt Express. 2005;13: 3252-3258.

11. Ikuno Y, Maruko I, Yasuno Y, et al. Reproducibility of retinal and choroidal thickness measurements in enhanced depth imaging and high-penetration optical coherence tomography. Invest Ophthalmol Vis Sci. 2011;52:5536-5540.

12. Ikuno Y, Jo Y, Hamasaki T, Tano Y. Ocular risk factors for choroidal neovascularization in pathologic myopia. Invest Ophthalmol Vis Sci. 2010;51:3721-3725

13. Leung CK, Cheng AC, Chong KK, et al. Optic disc measurements in myopia with optical coherence tomography and confocal scanning laser ophthalmoscopy. Invest Ophthalmol Vis Sci. 2007;48:3178-3183.
14. Saka N, Moriyama M, Shimada N, et al. Changes of axial length measured by IOL master during 2 years in eyes of adults with pathologic myopia. Graefes Arch Clin Exp Ophthalmol. 2013;251:495-499.

15. Margolis R, Spaide RF. A pilot study of enhanced depth imaging optical coherence tomography of the choroid in normal eyes. Am JOphthalmol. 2009; 147:811-815.

16. Ikuno Y, Kawaguchi K, Nouchi T, Yasuno Y. Choroidal thickness in healthy Japanese subjects. Invest Ophthalmol Vis Sci. 2010;51: 2173-2176.

17. Sadler TW. Langman's Medical Embryology, 10th ed. Baltimore: Lippincott Williams \& Wilkins; 2006.

18. Hayreh SS. In vivo choroidal circulation and its watershed zones. Eye. $1990 ; 4: 273-289$
Clinical Ophthalmology

\section{Publish your work in this journal}

Clinical Ophthalmology is an international, peer-reviewed journal covering all subspecialties within ophthalmology. Key topics include: Optometry; Visual science; Pharmacology and drug therapy in eye diseases; Basic Sciences; Primary and Secondary eye care; Patien Safety and Quality of Care Improvements. This journal is indexed on

Submit your manuscript here: http://www.dovepress.com/clinical-ophthalmology-journal

\section{Dovepress}

PubMed Central and CAS, and is the official journal of The Society of Clinical Ophthalmology (SCO). The manuscript management system is completely online and includes a very quick and fair peer-review system, which is all easy to use. Visit http://www.dovepress.com/ testimonials.php to read real quotes from published authors. 